\title{
LORENTZ TRANSMISSION ELECTRON MICROSCOPY INVESTIGATION OF MAGNETICALLY PATTERNED Co/Pt MULTILAYERS
}

\author{
G.J. KUSINSKI ${ }^{1,2}$, K.M. KRISHNAN ${ }^{2}$, D. WELLER ${ }^{3}$, B.D. TERRIS ${ }^{3}$, \\ L. FOLKS ${ }^{3}$, A.J. KELLOCK ${ }^{3}$, J.E.E. BAGLIN ${ }^{3}$ AND G. THOMAS ${ }^{1,2}$ \\ 1) Department of Materials Science and Engineering, University of \\ California, Berkeley, CA 94720, \\ ${ }^{2)}$ Materials Sciences Division, Lawrence Berkeley National Laboratory, \\ University of California, Berkeley, CA 94720 , \\ ${ }^{3)}$ IBM Almaden Research Center, 650 Harry Road, San Jose, CA 95120
}

\begin{abstract}
The switching behavior of magnetic patterns prepared by ion irradiation was investigated. $\mathrm{Co} / \mathrm{Pt}$ multilayers with perpendicular anisotropy and large out-of-plane coercivities 5-6 kOe were grown on electron transparent SiN windows. Regularly spaced 1 micron sized regions, were magnetically pattered via ion beam irradiation through a stencil mask. Lorentz TEM was used to observe in-situ magnetization reversal processes of irradiated regions under well-defined applied magnetic fields. When the in-plane field was increased, domain wall motion was observed, resulting in the alignment of the patterns with the direction of the applied field. The switching mechanism of the in-plane patterns was by domain wall motion.
\end{abstract}

\section{Introduction}

One of the approaches to ultrahigh-density magnetic storage is patterned magnetic media. Previously, small isolated islands of magnetic material have been produced by a number of techniques [1], all of which require a resist step and subsequent deposition or etch step. However, with the head-to-disk spacing in the range of $10 \mathrm{~nm}$, the cleanliness of the media surfaces is a key parameter. For this reason, it is desirable to develop a non-contact magnetic patterning method that preserves surface smoothness and planarity. Ion-beam patterning of magnetic thin films through suitable non-contact stencil masks, has been suggested 22 as a good candidate to produce such a planar patterned media. Co/Pt multilayers, in particular, are ideally suited for this application, since bombardment with a suitable dose of energetic ions has been shown to change the magnetic easy axis of the multilayer 3. The irradiation may induce interface mixing [4,5], thereby triggering a spin-reorientation transition from the out-of-plane to the inplane easy axis. In addition, ion-irradiation through a resist layer [6], particularly through non-contact stencil masks 7], were shown to produce a patterned magnetic film. Understanding the switching mechanism of sub-micron magnetic elements is an essential step for the implementation of such patterned media into data storage, and this is discussed in this paper. 


\section{Experimental}

The samples under study were $\mathrm{Co} / \mathrm{Pt}$ multilayers deposited by electron beam evaporation. The Co/Pt multilayer structure consisted of a $20 \mathrm{~nm} \mathrm{Pt}$ buffer layer, 10 periods of $3 \AA \mathrm{Co} / 10 \AA \mathrm{Pt}$, followed by a $10 \AA \mathrm{Pt}$ capping layer. Samples were grown on electron transparent $\mathrm{Si}_{3} \mathrm{~N}_{4}$ windows to facilitate transmission electron microscopy (TEM) and, in particular, the Lorentz magnetic imaging. Magnetic measurements of the as-grown samples were performed using polar and transverse Kerr hysteresis measurements.

As mentioned before, ion-irradiation was found to cause a rotation of the easy axis of the $\mathrm{Co} / \mathrm{Pt}$ multilayers to in-plane. The origin of such spin-reorientation (SR) induced by ion-irradiation is currently investigated by HRTEM but will not be discussed here. The ion induced SR was used to locally change the magnetization of the multilayer. Regularly spaced 1 micron sized regions were patterned via ion beam irradiation through a stencil mask with 1 micron sized circular holes. The mask was placed directly on the film and the sample was bombarded with $700 \mathrm{keV} \mathrm{N}^{+}$ions from a NEC 3UH Pelletron accelerator. The detailed description of this experiment is described elsewhere [7].

Lorentz transmission electron microscopy was used to investigate the magnetic structure of these patterns. The objective lens of the microscope is a source of vertical field, which can be controlled by adjusting the objective lens current [8]. In-situ magnetizing experiments were performed by applying a constant 1500 Oe vertical field. By tilting the sample up to $\pm 35^{\circ}$, an in-plane component of the field, perpendicular to the specimen tilt axis, can be applied. Sample was taken through the hysteresis loop, and the Lorentz Fresnel imaging was used to in-situ record the magnetization reversal processes of the patterned areas. In the Fresnel imaging mode contrast arises when there is a spatially varying component of the in-plane magnetic induction. Hence, domain walls appear as dark or bright fringes $[9,10]$.

\section{Results and Discussion}

Figure 1 shows a plan-view, bright field TEM image and an electron diffraction pattern of a $\mathrm{Co} / \mathrm{Pt}$ multilayer grown at $250^{\circ} \mathrm{C}$. The sample was polycrystalline with an average grain size of $50 \pm 16 \mathrm{~nm}$. The fine structure visible in some of the grains is attributed to Moiré fringes caused by the small lattice mismatch and rotation between Co and Pt planes. The films have a [111] texture, as indicated by the bright (220) ring in the selected area diffraction pattern (SADP) (see inset Figure 1). Figure 2 is a TEM cross-section image of the $\mathrm{Co} / \mathrm{Pt}$ multilayer. The grain growth is typically columnar with grains propagating through the film thickness.

Figure 3 shows a perpendicular hysteresis loop measured by Kerr rotation. The squareness of the loop indicates that the easy direction of magnetization is out-of-plane. High resolution TEM imaging found the multilayers to have a face centered cubic stacking, what implies a small volume anisotropy of Co. Hence, the perpendicular easy axis of magnetization is attributed to the interface anisotropy [5].

The deflection of electrons in the TEM is governed by the well-known Lorentz force,

$$
\mathbf{F}=-\mathrm{e}(\mathbf{v} \times \mathbf{B})
$$

where e is electron charge, $\mathbf{v}$ is the velocity of electrons and $\mathbf{B}$ is the magnetic induction. The deflection $\beta$ of electron is given by: 


$$
\beta=\mathrm{e} \cdot \lambda \cdot \mathrm{B}_{\perp} \mathrm{t} / \mathrm{h}
$$

where $h$ is Plank's constant, $\lambda$ is the electron wavelength, and $B_{\perp} \cdot t$ is the component of magnetic induction perpendicular to the electron path and integrated over thickness $t$. Therefore, the deflection of the beam is perpendicular to the magnetization. In the case of a uniformly magnetized "particle", this gives an increased intensity, or (seen as) bright fringe, at one end of the patterned region, and decreased intensity, or (seen as) dark fringe at the other end, as schematically illustrated in Figure 4. When magnetization is reversed the contrast is opposite.

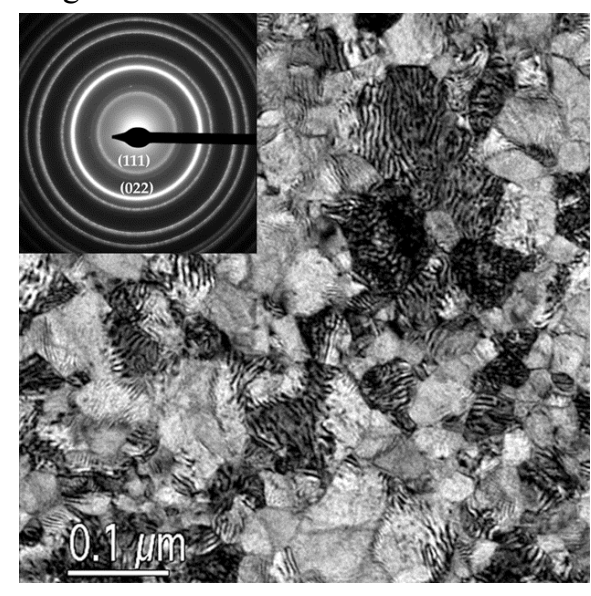

Figure 1. Transmission electron micrograph and selected area diffraction pattern of the $\mathrm{Co} / \mathrm{Pt}$ multilayer (see text).

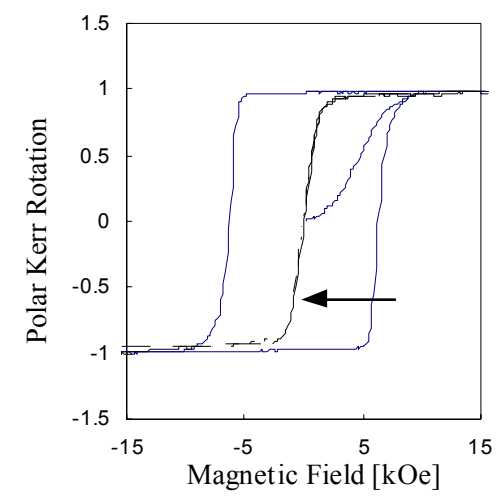

Figure 3. Polar Kerr hysteresis loops of a 10x $(3 \AA$ $\mathrm{Co} / 10 \AA \mathrm{Pt}$ ) multilayer. Arrow shows the loop for the irradiated sample.

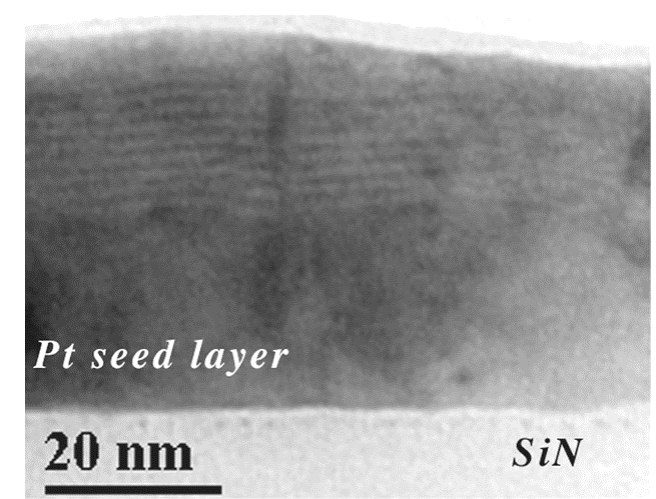

Figure 2. TEM cross-section image of the $\mathrm{Co} / \mathrm{Pt}$ multilayer showing columnar grain growth. Image slightly out of focus to enhance the ML's contrast.

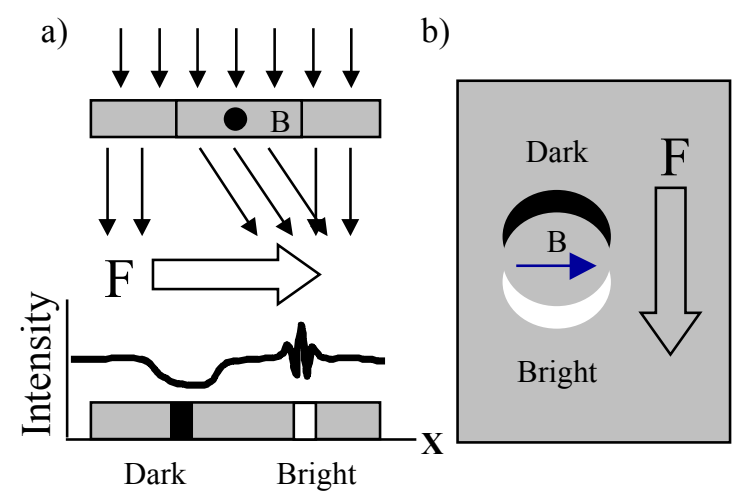

Figure 4. Schematic illustrating observed Fresnel contrast for patterned areas. a) side view, b) top view.

The analyzed multilayers consist of only $3 \mathrm{~nm}$ of magnetic thickness, what results in a very small magnetic signal contribution to the overall contrast. This is mainly due to the strong microstructure contrast, arising from the diffraction of the individual columnar grains, as shown in Figure 1. However, in this case the physical microstructure is on a significantly smaller scale than the magnetic pattern size $(50 \mathrm{~nm}$ 
vs. $1 \mu \mathrm{m}$ ). Hence, the influence of the high spatial frequency component (physical microstructure) was reduced by utilizing a large $100 \mu \mathrm{m}$ defocus in the Fresnel mode, which however limits the resolution.

Figure 5 shows a series of Fresnel images of the patterned multilayers, recorded at different sample tilts, and hence at a different in-plane magnetic field applied. For each image, the sample tilt and the applied in-plane magnetic field are indicated. Figure 5a is an image recorded at a $10^{\circ}$ tilt $\left(\mathrm{H}_{\|}=264 \mathrm{Oe}\right)$. This field was sufficient to align all the in-plane patterned areas. The Fresnel contrast consists of dark and bright fringes at the top and bottom, respectively, of each irradiated pattern. The contrast is consistent with the sample being magnetized to the right, along the applied field direction as indicated by the arrow and explained in Figure 4.
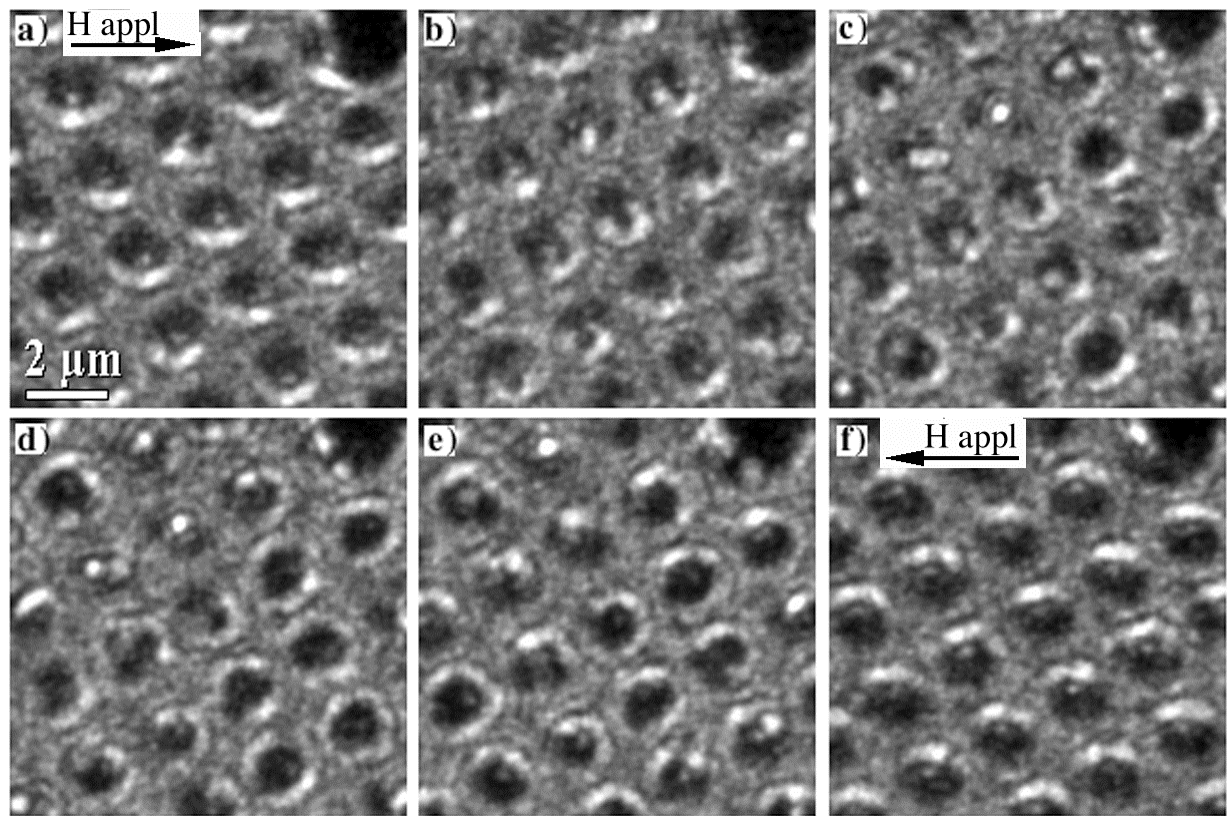

Figure 5. A series of Fresnel images of patterned multilayers recorded at different sample tilts.

a) tilt $\left.=10^{\circ} ; \mathrm{H}_{||}=264 \mathrm{Oe}, \mathrm{b}\right)$ tilt $\left.=0.3^{\circ} ; \mathrm{H}_{||}=7 \mathrm{Oe}, \mathrm{c}\right)$ tilt $\left.=-2^{\circ} ; \mathrm{H}_{||}=-54 \mathrm{Oe}, \mathrm{d}\right)$ tilt $=-3.1^{\circ} ; \mathrm{H}_{||}=-82 \mathrm{Oe}$, e) tilt $\left.=-5.1^{\mathrm{o}} ; \mathrm{H}_{||}=-135 \mathrm{Oe}, \mathrm{f}\right)$ tilt $=-10^{\circ} ; \mathrm{H}_{||}=-264 \mathrm{Oe}$.

When the in-plane field was decreased, the sample remained magnetized in the same direction. Figure 5b, shows the area with the field reduced to $\mathrm{H}_{\|}=\sim 7$ Oe. Patterned areas still have a white fringe at the bottom but some develop split contrast. When the field was applied in negative direction, reversal was observed by domain wall motion. The reversal, for some of the patterns, starts at relatively low fields (Figure $5 \mathrm{c}$, $\mathrm{H}_{\|}=-54 \mathrm{Oe}$ ), however, patterns magnetized in positive direction are still present. At this field for some of the patterns, the contrast changes to a bright (dark) fringe at the top and at the bottom what corresponds to white, almost continuous, circular fringe and dark center, (dark area with bright middle). The patterns showing this contrast support the multi-domain state, and hence Lorentz deflection is in both directions. When the field is further increased in negative direction, (Figure $5 \mathrm{~d}, \mathrm{H}_{\|}=-82 \mathrm{Oe}$ ), the multidomain contrast is observed for most of the patterns. As the field is further increased, 
(Figure $5 \mathrm{e}, \mathrm{H}_{\|}=-135 \mathrm{Oe}$ ), the observed contrast is similar to that in Figure $5 \mathrm{~d}$, however notice that the bright fringes are less continuous, and are brighter at the top of the patterns what implies multidomain state with majority of the area already magnetized in negative direction. Finally, when $\mathrm{H}_{\|}=-264 \mathrm{Oe}$ is applied further domain wall motion was observed. This resulted in the complete alignment of the patterns with the direction of the applied field, (to the left), as shown in Figure 5f. The Fresnel contrast consists only of dark and bright fringes at the bottom and the top, respectively, which is opposite to the contrast shown on Figure 5a.

\section{Conclusions}

The analyzed $\mathrm{Co} / \mathrm{Pt}$ multilayers were polycrystalline [111] textured, with an average grain size of $50 \pm 16 \mathrm{~nm}$. The switching mechanism of the in-plane patterns prepared by ion irradiation was by domain wall motion. In the remanent state, and with no field applied, the irradiated areas were in-plane and supported a multi-domain configuration. The significant softening of the in-plane regions $\left(\mathrm{H}_{\mathrm{c}}<300 \mathrm{Oe}\right)$ as compared to the out-of-plane coercivity was recorded.

\section{Acknowledgements}

Greg Kusinski acknowledges financial support through the "IBM Research Fellowship". Work at MSD and NCEM at LBNL was supported by the Director, Office of Energy Research, Office of Basic Energy Sciences, Materials Sciences Division of the U.S. Department of Energy under contract No. DE-AC03-76SF00098

\section{References}

1 Farhoud, M.; Hwang, M.; Smith, Henry I.; Schattenburg, M.L.; Bae, J.M.; Youcef-Toumi, K.; Ross, C.A. (1998) Fabrication of large area nanostructured magnets by interferometric lithography, IEEE Transactions on Magnetics 34, 1087-1089.

Ross, CA; Smith, HI; Savas, T; Schattenburg, M; Farhoud, M; Hwang, M; Walsh, M; Abraham, MC; Ram, RJ. (1999) Fabrication of patterned media for high density magnetic storage, J. Vac. Sci. Tehcnol. B 17, 3168-3176.

2 Chappert, C., Bernas, H., Ferré, J., Kottler, V., Jamet, J. -P., Chen, Y., Cambril, E., Devolder, T., Rousseaux, F., Mathet, V., and Launois, H. (1998) Planar Patterned Magnetic Media Obtained By Ion Irradiation, Science 280, 1919-1922.

3 Ferré, J., Chappert, C., Bernas, H., Jamet, J.-P., Meyer, P., Kaitasov, O., Lemerle, S., Mathet, V., Rousseaux, F., and Launois H. (1999) Irradiation Induced Effects on Magnetic Properties of $\mathrm{Pt} / \mathrm{Co} / \mathrm{Pt}$ Ultrathin Films, J. Magn. Magn. Mater. 191,198-199.

4 Carcia, P.F., Shah, S.I., and Zeper, W.B. (1990) Effect of energetic bombardment on the magnetic coercivity of sputtered Pt/Co thin-film multilayers, Appl. Phys. Lett. 56, 2345.

5 Weller, D. Baglin, J.E.E., Kellock, A.J., Hannibal, K.A., Toney, M.F., Kusinski, G., Lang, S., Folks, L., Best M.E., and Terris, B.D. (2000) Ion Induced Magnetization Reorientation in Co/Pt Multilayers for Patterned Media, J. Appl. Phys. 87, 5768-5770.

6 Devolder, T., Chappert, C., Chen, Y., Cambril, E., Bernas, H., Jamet, J.-P., and Ferré, J. (1999) Sub 50 nm planar magnetic nanostructures fabricated by ion irradiation, Appl. Phys. Lett. 74, 3383-3385.

7 Terris, B., Folks, L., Weller, D., Baglin, J.E.E., Kellock, A.J., Rothuizen, H., and Vettiger, P. (1999) Ion Beam Patterning of Magnetic Films Using Stencil Masks, Appl. Phys. Lett. 75, 403-405.

8 Verbist, K., Nelson, E.C., Anthony, T.C., Brug, J.A., and K.M. Krishnan (1998) Lorentz transmission electron microscopy in a standard CM200FEG, Proc. Int. Conf. Elec. Mic., ICEM14, vol II, 503-504

9 Fuller, H.W., and Hale, M.E. (1959) Determination of magnetization distribution in thin foils using electron microscopy, J. Appl. Phys. 31, 238-234.

10 Chapman, J.N. (1984) The investigation of magnetic domain structures in thin foils by electron microscopy, J. Phys. D: Apply. Phys. 17, 623-647 